\title{
Overview on Parkinson's disease: pathophysiology, and experimental models
}

\author{
Khalid Ahmad Mohamad", Sara A. Wahdan, Reem N. El-Naga \\ Department of Pharmacology and Toxicology, Faculty of Pharmacy, Ain Shams University, Cairo 11566, Egypt
}

\begin{abstract}
Parkinson's disease, a neurodegenerative disease, is caused by dopaminergic neurons death and accompanied by rigidity, and postural instability, as well as bradykinesia. The cause of these neurons' death is still unclear. Since the dopaminergic neurons couldn't regenerate, therefore Parkinson's disease couldn't be cured. Thus, over the past decades, significant effort has been made to explore the etiology of Parkinson's disease development and ascertainment. This review aimed to highlight the progress that has been made in understanding Parkinson's disease pathophysiology. The role of oxidative stress, neuroinflammation, and apoptosis in the development of PD has been discussed. It has been noticed that oxidative stress, inflammation, and apoptosis are working together to develop Parkinson's disease, and each of these factors affects each other. Additionally, the experimental models and their drawbacks have been emphasized. Additionally, the mechanism of inducing Parkinson's disease (i.e., inducing neuroinflammation and oxidative stress) by neurotoxin has been highlighted.
\end{abstract}

Keywords: Parkinson's disease; epidemiology; pathophysiology; oxidative stress; neuroinflammation; experimental models.

*Correspondence | Khalid Ahmad Mohamad; Department of Pharmacology and Toxicology, Faculty of Pharmacy, Ain Shams University, Cairo, Egypt. Email: khaled.mohamed1@pharma.asu.edu.eg

Citation | Mohamad KA, Wahdan SA, El-Naga RN, 2021. Overview on Parkinson's disease: pathophysiology, and experimental models. Arch

Pharm Sci ASU 5(2): 212-224

DOI: $10.21608 /$ aps.2021.92639.1068

Print ISSN: 2356-8380. Online ISSN: 2356-8399.

Received 30 August 2021. Accepted 06 September 2021.

Copyright: ${ }^{\circledR} 2021$ Mohamad et al. This is an open-access article licensed under a Creative Commons Attribution 4.0 International License (CC BY 4.0), which permits unrestricted use, distribution, and reproduction in any medium, provided the original author(s) and source are credited.

Published by: Ain Shams University, Faculty of Pharmacy

\section{Introduction}

Parkinson's disease (PD) is second to Alzheimer's disease as the most prevalent neurodegenerative disorder throughout the world [1]. Paralysis agitans was first medically described as a neurological syndrome by $\mathrm{J}$. Parkinson in the $19^{\text {th }}$ century in the book "Essay on the Shaking Palsy" and renamed PD by JeanMartin Charcot [2, 3].

Clinically, PD is characterized by motor dysfunction, i.e., rest tremors, bradykinesia, postural instability, and rigidity. Also, psychiatric symptoms are involved, including depression and anxiety, as well as autonomic dysfunction manifestoes, i.e., constipation and hypotension. Besides, it is characterized by paresthesia, olfactory dysfunction, and sleep disorders [4]. A review of incidence studies reported that the PD incidence rises with age to a peak occurring in the seventh decade of life and it continues to rise after this age in some studies [5].

Pathologically, PD is associated with Lewy bodies (LBs), dopaminergic neuronal cytoplasmic inclusions. These are the hallmarks of PD, as they are not detectable in healthy individuals. In 1997, LBs were reported to contain aggregates of $\alpha$-synuclein ( $\alpha$-Syn) [6].

In this review, the pathogenesis of $\mathrm{PD}$, and 
neurotoxin models will be discussed.

\section{The pathophysiology of PD}

Pathologically, PD is characterized by hallmarked dopaminergic neurons lost in the substantia nigra and the appearance of LBs that are primarily comprised of fibrillar $\alpha$-Syn [7].

Genetically, studies on familial PD have identified mutations in single genes in monogenic PD. In particular, mutations, which give rise to $\mathrm{PD}$, are located in the $\alpha$-Syn-encoded genes, dardarin, vacuolar protein sorting-associated protein 35, parkin ligase, deglycase DJ1, and acid $\beta$-glucosidase [8].

Despite mutations in these genes being infrequent and only exist in less than a tenth of all PD cases [9], they have discovered key processes and molecular players in the etiology of PD. This can be clarified by the gene (SNCA), which is linked to PD at a neuropathological and genetic level. Additionally, LBs and $\alpha$-Syn are recognized in familial- and idiopathic- PD. Besides the mutations in SNCA and copy number variations present in monogenic PD [10], common SNCA mutations are linked to an increased risk of idiopathic PD [11].

Because there is no monogenic inheritance pattern in most PD patients (90\%), the disease is considered idiopathic. Sporadic PD has a multiple-factorial etiology; environmental and genetic factors work together to determine an individual's liability to disease $[\mathbf{1 2}, \mathbf{1 3}]$.

\subsection{The role of oxidative stress in PD}

Mounting evidence has pointed that oxidative damage and mitochondrial dysfunction result in a cascade of events and eventually contribute to dopaminergic neurons degeneration [14]. This notion was supported by the assessment of the postmortem brain sections, in which elevated levels of 4-hydroxy-2-nominal, a lipid peroxidation by-product [15], carbonyl modifications of soluble proteins [16], and oxidation products of DNA, i.e., 8-hydroxydeoxyguanosine and RNA, i.e., 8-hydroxyguanosine $[17,18]$ have been detected.

In the presence of oxygen, metals, or enzymes, such as tyrosinase, dopamine (DA) are oxidized and form free radicals and quinones [19]. Dopamine quinones form a monochrome, a cyclic highly reactive, and cause the production of superoxide and reduction in cellular NADPH. A monochrome can also induce neuroinflammation by neuroinflammation, as it is the precursor of neuromelanin [19].

Postmortem tissues from the brain of PD patients have shown depletion in the amount of glutathione (GSH) in substantia nigra compacta compared to the controls [20]. Glutathione is generated in the cytoplasm but it is transported to the mitochondria to work as an anti-oxidant [21]. Since apoptosis is induced by oxidative stress, the mitochondrial GSH is considered a crucial marker of oxidative stress assessment. Also, mounting observations indicated that complex-I dysfunction causes higher production of ROS and subsequently a reduction in GSH. This reduction can result from a decreased synthesis of GSH by the suppressing of glutathione reductase, or rising glutathione disulfide level [22, 23]. Oppositely, reduced GSH level leads to complex-I activity impairment, and overall mitochondrial function [24].

Iron is pivotal for most human cells. Indeed, it is a cofactor for important proteins to maintain the normal neurons function, like tyrosine hydroxylase, an important enzyme for the synthesis of neurotransmitters [25]. Iron can contribute to ROS generation by the reaction of ferric/ferrous with superoxide anion radical, and hydrogen peroxide, producing the hydroxyl radicals, which trigger neurotoxicity with DA oxidation [26]. 


\subsection{The role of inflammation in PD pathogenesis}

The inflammatory process, which is a protective mechanism against any infection, damage, or injury, is mediated by several immune cells, i.e., microglia, neutrophils, and macrophages [27, 28]. Unlike other cells, damaged neurons can't regenerate [29]. The acute inflammatory response assists to fight the toxins, infectious agents, phagocytose cellular debris, and repair the affected tissues [30]. However, for a prolonged duration, the inflammatory response could be destructive, i.e., it prevents repairing of tissue, and regeneration. Thus, chronic inflammation magnifies the neurodegeneration progression [30].

The implication of neuroinflammation in PD pathology is based on quite a lot of research of evidence suggesting that neuro-inflammatory processes could possess a causal role in PD development [31]. Also, inflammation is suspected to be a melting point for genetic and also environmental factors that provoke PD pathogenesis [7].

Microglia constitute about a tenth of all glia and are usually resting in the adult brain playing beneficial housekeeping roles, as removal of toxic substances, synaptic remodeling, neuronal repair, and synaptic pruning [32]. Environmental challenges, morphological changes, intracellular molecules, and surface antigens could provoke microglial activation [33]. $\alpha$-Syn and soluble molecules liberated from dying neurons could also activate the microglia [33]. In the activation state, the microglia up-regulate many receptors implicated in inflammation, and could also generate potential neurotoxins, i.e., superoxide anions [34].

Similar to microglia, the astrocytes possess primarily neuroprotective effects, associated with GSH release and scavenging of excitotoxic agents, like glutamate and calcium [35, 36]. Recent studies have linked astrogliosis with the development of PD [37]. The astrocytes and endothelial cells together with pericytes form the blood-brain barrier (BBB), which isolates the CNS from the peripheral circulation [38]. The breakdown of BBB and increase in its permeability result in secondary leukocytes movement within the brain parenchyma, reactive gliosis, and damaged neurons [39]. Thus, the immune cells invade the parenchyma of the brain and eventually induce degeneration of neurons [39].

The postmortem analyses showed accumulation of pro-inflammatory cytokines, i.e., TNF- $\alpha$, and IL- 6 , in cerebrospinal fluid and brains of PD patients, which confirms progressing neuroinflammation $[\mathbf{4 0}, 41]$. The serum IL-6, Normal $T$ cell Expressed and Secreted (RANTES), and the chemokine ligand 5 (CCL5) are considerably increased in PD patients $[42,43]$. The intensity of the disease is correlated with serum RANTES levels [42].

A high nitric oxide synthase level has been observed in PD patients [44] indicating that cytokines-stimulated toxicity and inflammationstimulated oxidative stress could be implicated in the neurodegeneration, and disease ascertainment [45].

\subsection{The role of apoptosis in PD pathogenesis}

Apoptosis starts with specific internal/external signals and plays a substantial role in aging, neoplasm, and neurological disorders [46]. Based on the postmortem recognition of fragmentation of DNA, and apoptotic chromatin alterations in dopaminergic neurological cells of PD suffer, it is evident that apoptosis is the primary mechanism of neurons demise in PD [47].

Additionally, the implication of apoptosis in 
PD etiology has been confirmed in postmortem, in vivo, and in vitro studies that revealed increased apoptosis markers in Substantia nigra compacta [48-51]. Despite the extrinsic apoptotic pathway could be implicated in PD, the intrinsic dopaminergic neurons apoptosis pathway is still believed to be the predominant [52].

Several inherited forms of PD develop because of genes (LRRK2, PINK1, and DJ-1) mutations related to mitochondrial function [53]. Although these mutations are uncommon within the PD patients, they provide some supporting evidence to the susceptibility of mitochondria damage-mediated apoptosis, and to these relevant processes in idiopathic PD [53].

Parkin possesses many roles that could be relevant in PD pathogenesis, as it can induce mitochondrial- biogenesis, genes transcription, and DNA replication [54]. Furthermore, Parkin performs as an E3 ubiquitin-protein ligase involved in the degradation by the ubiquitinproteasome system, i.e., the glycosylated form of $\alpha$-Syn [55]. The impairment in Parkin activity is believed to increase protein aggregates causing PD [55].

Additionally, mutations in leucine-rich repeat kinase 2 (LRRK2) have been considered as the main cause of hereditary PD [56] and can lead to defective dynamic/morphology of mitochondria, and exaggerate generation of ROS [57]. These mutations are also proposed to cause neuronal demise by apoptosis caused by mitochondrial malfunction. Cell death could be experimentally triggered by the up-regulation of mutant LRRK2 with apoptosis being inhibited by caspase inhibitors [58].

Additionally, DNA removals have been previously reported in dopaminergic neuronal cells in both elderly and PD suffers, possibly heightening their vulnerability to apoptosis [59]. The anticipatory mechanisms of DNA removals are unidentified with the possibility of oxidative stress involvement [60]. Depletion and deletion in mitochondrial DNA lead to a decrease in the performance and integrity of mitochondria, consequently exaggerating the release of cytochrome c and apoptosis [61]. Furthermore, a rare type of inherited PD could develop because of variation in POLG, a gene that is involved in the expression of many genes encoded in mitochondrial DNA [62].

\section{Neurotoxin-based model of PD}

Epidemiological studies have affirmed that prolonged exposure to rural chemicals, i.e., paraquat and rotenone elevate the risk of PD development [63]. Dopamine structural analogs, like MPTP, and 6-hydroxydopamine (6-OHDA), have been reported to selectively damage dopaminergic neurons and induce parkinsonism.

\subsection{The 6-hydroxydopamine model of PD}

6-Hydroxydopamine is the first neurotoxin used to induce PD because it could induce mitochondrial dysfunction of dopaminergic neurons [64]. Since 6-OHDA cannot cross BBB, it has to be injected intra-cerebrally. 6Hydroxydopamine is transferred into the neurons by the DA transporter and then suppresses the activity of mitochondrial respiratory chain complex-I $[65,66]$. In mitochondrial, 6-OHDA can also suppress the complex-IV activity and reduce membrane potential [67].

Once inside the neurons, 6-OHDA produces $\mathrm{H}_{2} \mathrm{O}_{2}$, and superoxide from its metabolic degradation or oxidation. ROS cause protein- and DNA oxidation, and lipid peroxidation, and eventually lead to oxidative stress and mitochondrial impairment $[68,69]$. 6Hydroxydopamine could increase glutamate, and lower the striatal glutamine, resulting in an imbalance between excitatory and inhibitory brain processes, causing long-term irregularities in activities of the glutamate system and 
GABAergic system [70].

The severity of symptoms induced depends on the injection site, as 6-OHDA administration into the striatum induces relatively mild symptoms with a slow progression; while, the direct 6-OHDA administration into the medial forebrain and $\mathrm{SN}$, induces severe symptoms with fast and significant severe dopaminergic cell death [71]. However, the 6-OHDA-based model lacks Lewy pathology [72].

\subsection{1-Methyl-4-Phenyl-1, 2, 3, 6- Tetrahydropyridine (MPTP) model of PD}

The PD model induced by MPTP is an experimental model based on the systemic treatment of MPTP, which has a high toxically affinity to dopaminergic neurons [73]. Langston et al. have described parkinsonism in a group of drug abusers mediated by intravenous injection of MPTP with an illegal neurotoxin-containing drug [74, 75].

After crossing the BBB, MPTP is converted by MAO-B into 1-methyl-4-phenylpyridinium ion $\left(\mathrm{MPP}^{+}\right)$, its active form, and then carried by the DA transporter inside the dopaminergic neurons, where it suppresses the mitochondrial complex-I activity [76]. It has been re-assessed following MPTP treatment through the intranasal route, resulting in depletion of striatal DA, accompanied by PD symptoms [77].

The main limitation of the MPTP-based model is that it lacks Lewy pathology. Therefore, myriads of studies have attempted to solve the missing of this crucial neuropathological PD hallmark by changing treatment regimens. It has been induced of ubiquitin and $\alpha$-Syn formation by a 30-day administration of MPTP via osmotic minipumps [78]; while Shimoji et al. has not succeeded to detect LBs in mice treated with different regimens of MPTP treatment without using osmotic minipumps [79].

Additionally, the dopaminergic nigrostriatal deficits resulting from acute or sub-acute administration of MPTP are reversible, however, the chronic coadministration of MPTP and probenecid has been demonstrated to overcome this limitation [80].

\subsection{The paraquat model of $P D$}

Paraquat is an herbicide that has been got great interest due to its chemical structural similarity to $\mathrm{MPP}^{+}[\mathbf{8 1}]$. Paraquat has been used to induce the PD model as it can penetrate the BBB by the neutral amino acid transporter since the L-valine treatment significantly reduced the paraquat penetration of $\mathrm{BBB}$ [82]. Paraquat impairs the redox recycling of GSH and thioredoxin inducing oxidative stress [83].

However, paraquat administration causes acute toxicity in many organs, particularly lung tissues; thus, it may affect motor performance and cause a high mortality rate [84] Additionally, the use of the paraquat model in examining neuroprotective therapies is limited due to the lack of paraquat-induced striatal DA depletion [85].

\subsection{The rotenone model of PD}

Rotenone is an isoflavone found in the roots and stems of the Lonchocarpus and Derris, and due to its high lipophilicity, it can cross BBB [86]. As soon as ROT is in the dopaminergic neurons, it inhibits the complex-I activity, resulting in an elevation in ROS production and mitochondrial dysfunction [87]

Lipid and also glutamine metabolism alterations by rotenone play a pivotal compensatory role in PD modeling [88]. ROT has become of high interest following the seminal paper by the Greenamyre group in 2000, it was continuously IV infused into the back of Lewis rats at a concentration of $3 \mathrm{mg} / \mathrm{kg} /$ day [89]. Rotenone induces $\alpha$-Syn accumulation and aggregation replicating the neuropathological hallmark of LBs seen in PD [51]; another reason 
for the ROT-based model of PD to outweigh other neurotoxin-based models and qualify this model to closely simulate human PD.

The main limitations of the ROT-based model are low reproducibility in the animals, i.e., they develop varied dopaminergic lesions, size and location of lesions, and mortality [90]. These limitations have been overcome to some extent by using different routes of ROT administration [86].

\section{Conclusion}

Significant advances in understanding the pathogenesis of PD have been concluded from the epidemiological findings, experimental methods, and pathological manifestations. Signaling pathways have been detected, accompanied by mitochondrial homeostasis impairments, and protein accumulation, and are likely to be involved in PD etiology. Additionally, a substantial advance has been made in PD modeling. However, each model has advantages and limitations. Further research is needed to develop the currently used models or new models to recapitulate the human PD.

\section{List of abbreviations}

6-OHDA, 6-Hydroxydopamine; $\alpha$-Syn, $\alpha$ synuclein; BBB, blood-brain barrier; DA, Dopamine; GSH, Glutathione; LBs, Lewy bodies; MPP+, 1-Methyl-4-phenylpyridinium ion; MPTP, 1-Methyl-4-Phenyl-1,2,3,6Tetrahydropyridine; PD, Parkinson's disease; ROS, Reactive oxygen species; ROT, Rotenone

\section{Declarations}

\section{Ethics approval and consent to participate}

Not applicable

\section{Consent to publish}

All authors have read and agreed to the published version of the manuscript.

\section{Availability of data and materials}

Data analyzed during this study are all included in the main manuscript.

\section{Competing interests}

No competing interests were declared by the authors.

\section{Funding statement}

No funding source was received

\section{References}

1. Vial F, Delgado I, Idiaquez JF, Canals F, Chana-Cuevas P. Epidemiology of Parkinson's Disease in Chile. Neuroepidemiology. 2021:1-5 https://doi.org/10.1159/000517750.

2. Weiner WJ. There is no Parkinson's disease. Archives of neurology. 2008; 65(6): 705-8 https://doi.org/10.1001/archneur.65.6.705.

3. Frucht SJ, Termsarasab P. Typical Parkinson's Disease Phenomenology. Movement Disorders Phenomenology: Springer; $2020 . \quad$ p. $17-35$ https://doi.org/10.1007/978-3-030-36975-0_3.

4. Magrinelli F, Picelli A, Tocco P ,Federico A, Roncari L, Smania N, et al. Pathophysiology of motor dysfunction in Parkinson's disease as the rationale for drug treatment and rehabilitation. Parkinson's disease. 2016;2016 https://doi.org/10.1155/2016/9832839.

5. Twelves D, Perkins KS, Counsell C. Systematic review of incidence studies of Parkinson's disease. Movement disorders: official journal of the Movement Disorder Society. $\quad 2003 ; \quad 18(1)$ : 19-31 https://doi.org/10.1002/mds.10305.

6. Goedert M, Jakes R, Spillantini MG. The synucleinopathies: twenty years on. Journal of Parkinson's disease. 2017; 7(s1): S51-S69 https://doi.org/10.3233/JPD-179005. 
7. Tansey MG, Goldberg MS. Neuroinflammation in Parkinson's disease: its role in neuronal death and implications for therapeutic intervention. Neurobiology of disease. $\quad 2010 ; \quad 37(3): \quad 510-8$ https://doi.org/10.1016/j.nbd.2009.11.004.

8. Deng H, Wang P, Jankovic J. The genetics of Parkinson's disease. Aging research reviews. 2018; 42: $72-85$ https://doi.org/10.1016/j.arr.2017.12.007.

9. Ross OA. A prognostic view on the application of individualized genomics in Parkinson's disease. Current genetic medicine reports. 2013; 1(1): 52-7 https://doi.org/10.1007/s40142-012-0003-1.

10. Singleton A, Farrer M, Johnson J, Singleton A, Hague S, Kachergus J, et al. $\alpha$-Synuclein locus triplication causes Parkinson's disease. Science. 2003; 302(5646): 841https://doi.org/10.1126/science.1090278.

11. Farrer $M$, Maraganore DM, Lockhart $P$, Singleton A, Lesnick T, De Andrade M, et al. $\alpha$-Synuclein gene haplotypes are associated with Parkinson's disease. Human molecular genetics. 2001; 10(17): 1847-51 https://doi.org/10.1093/hmg/10.17.1847.

12. Nalls MA, Pankratz N, Lill CM, Do CB, Hernandez DG, Saad M, et al. Large-scale meta-analysis of genome-wide association data identifies six new risk loci for Parkinson's disease. Nature genetics. 2014; 46(9): 989 https://doi.org/10.1038/ng.3043.

13. Delamarre A, Meissner WG. Epidemiology, environmental risk factors, and genetics of Parkinson's disease. La Presse Médicale. 2017; 46(2): 175-81 https://doi.org/10.1016/j.lpm.2017.01.001.

14. Rani L, Mondal AC. Emerging concepts of mitochondrial dysfunction in Parkinson's disease progression: Pathogenic and therapeutic implications. Mitochondrion. 2020; $\quad 50: \quad 25-34$ https://doi.org/10.1016/j.mito.2019.09.010.

15. Haga H, Matsuo K, Yabuki Y, Zhang C, Han F, Fukunaga K. Enhancement of ATP production ameliorates motor and cognitive impairments in a mouse model of MPTPinduced Parkinson's disease. Neurochemistry international. 2019; 129: 104492 https://doi.org/10.1016/j.neuint.2019.104492.

16. Floor E, Wetzel MG. Increased protein oxidation in human substantia nigra pars compacta in comparison with basal ganglia and prefrontal cortex measured with an improved dinitrophenylhydrazine assay. Journal of neurochemistry. 19(1)70;98 7-268: https://doi.org/10.1046/j.14714159.1998.70010268.x.

17. Alam Z, Jenner A, Daniel S, Lees A, Cairns $\mathrm{N}$, Marsden $\mathrm{C}$, et al. Oxidative DNA damage in the parkinsonian brain: an apparent selective increase in 8-hydroxyguanine levels in substantia nigra. Journal of neurochemistry. 1997; $\quad$ 69(3): 1196-203 https://doi.org/10.1046/j.14714159.1997.69031196.x.

18. Zhang J, Perry G, Smith MA, Robertson D, Olson SJ, Graham DG, et al. Parkinson's disease is associated with oxidative damage to cytoplasmic DNA and RNA in substantia nigra neurons. The American journal of pathology. 1999; 154(5): 1423-9 https://doi.org/10.1016/S00029440(10)65396-5.

19. Delcambre S, Nonnenmacher Y, Hiller K. Dopamine metabolism and reactive oxygen species production. Mitochondrial Mechanisms of Degeneration and Repair in Parkinson's Disease: Springer; 2016. p. 25-47 https://doi.org/10.1007/978-3-319-42139-1.

20. Wang T, Li C, Han B, Wang Z, Meng X, 
Zhang L, et al. Neuroprotective effects of Danshensu on rotenone-induced Parkinson's disease models in vitro and in vivo. BMC Complementary Medicine and Therapies. 2020; 20(1): $1-10$ https://doi.org/10.1186/s12906-019-2738-7.

21. Smith GA, Lin T-H, Sheehan AE, van Naters WVdG, Neukomm LJ, Graves HK, et al. Glutathione S-Transferase regulates mitochondrial populations in axons through increased glutathione oxidation. Neuron. 2019; 103(1): 52-65. e6 https://doi.org/10.1016/j.neuron.2019.04.017.

22. Genestra M. Oxyl radicals, redox-sensitive signaling cascades, and antioxidants. Cellular signaling. $\quad 2007 ; \quad 19(9)$ : 1807-19 https://doi.org/10.1016/j.cellsig.2007.04.009.

23. Tian Y, Lu J, Hao X, Li H, Zhang G, Liu X, et al. FTH1 Inhibits Ferroptosis Through Ferritinophagy in the 6-OHDA Model of Parkinson's Disease. Neurotherapeutics. 2020:1-17 https//:doi.org/10.1007/s13311020-00929-z.

24. Han L, Mei J, Ma J, Wang F, Gu Z, Li J, et al. Cinnamaldehyde induces endogenous apoptosis of the prostate cancer-associated fibroblasts via interfering with the Glutathione-associated mitochondria function. Medical Oncology. 2020; 37(10): 1-11 https://doi.org/10.1007/s12032-020-01417-2.

25. Georgieff MK. Iron deficiency in pregnancy. American journal of obstetrics and gynecology. 2020 https://doi.org/10.1016/j.ajog.2020.03.006.

26. Yu B, Wang Z, Almutairi L, Huang S, Kim M-H. Harnessing iron-oxide nanoparticles towards the improved bactericidal activity of macrophage against Staphylococcus aureus. Nanomedicine: Nanotechnology, Biology, and Medicine. 2020; 24: 102158 https://doi.org/10.1016/j.nano.2020.102158.

27. Chen J ,Zhang X, Zhang C, Wang W, Chen $\mathrm{R}$, Jiao $\mathrm{H}$, et al. Anti-inflammation of natural components from medicinal plants at low concentrations in the brain via inhibiting neutrophil infiltration after stroke. Mediators of inflammation. 2016; 2016 https://doi.org/10.1155.9537901/2016/

28. Krakauer T. Inflammasomes, autophagy, and cell death: the trinity of innate host defense against intracellular bacteria. Mediators of inflammation. 2019; 2019 https://doi.org/10.1155/2019/2471215.

29. Rodemer W, Hu J, Selzer ME, Shifman MI. Heterogeneity in the regenerative abilities of central nervous system axons within species: why do some neurons regenerate better than others? Neural Regeneration Research. 2020; 15(6): 996 https://doi.org/10.4103/16735374.270298 .

30. Snyder B, Shell B ,Cunningham JT, Cunningham RL. Chronic intermittent hypoxia induces oxidative stress and inflammation in brain regions associated with early-stage neurodegeneration. Physiological reports. 2017; $5(9)$ https://doi.org/10.14814/phy2.13258.

31. Salama RM, Abdel-Latif GA, Abbas SS, Hekmat M, Schaalan MF. Neuroprotective effect of crocin against rotenone-induced Parkinson's disease in rats: Interplay between $\mathrm{PI} 3 \mathrm{~K} / \mathrm{Akt} / \mathrm{mTOR}$ signaling pathway and enhanced expression of miRNA-7 and miRNA-221. Neuropharmacology. $202: 164 ; 0$ 107900

https://doi.org/10.1016/j.neuropharm.2019.10 7900 .

32. Li Q, Barres BA. Microglia and macrophages in brain homeostasis and disease. Nature Reviews Immunology. 2018; 18(4): 225 
https://doi.org/10.1038/nri.2017.125.

33. Von Bernhardi R, Eugenín-von Bernhardi L, Eugenín J. Microglial cell dysregulation in brain aging and neurodegeneration. Frontiers in aging neuroscience. 2015; 7: 124 https://doi.org/10.3389/fnagi.2015.00124.

34. Liu X, Yuan Q, Li G-x, Jia C-c, Liu J-y, Yang Y-q, et al. Regulation of Superoxide by BAP31 through Its Effect on p22phox and Keap1/Nrf2/HO-1 Signaling Pathway in Microglia. Oxidative medicine and cellular longevity. $\quad 2021 ; 2021$ https://doi.org/10.1155/2021/1457089.

35. Ma K, Ding X, Song Q, Han Z, Yao H, Ding $\mathrm{J}$, et al. Lactate enhances Arc/arg3. 1 expression through hydroxycarboxylic acid receptor 1- $\beta$-arrestin2 pathway in astrocytes. Neuropharmacology. 2020: 108084 https://doi.org/10.1016/j.neuropharm.2020.10 8084.

36. Misra UK, Singh SK, Kalita J, Kumar A. Astrocyte activation following nitrous oxide exposure is related to oxidative stress and glutamate excitotoxicity. Brain Research. 2020; 1730 : 146645 https://doi.org/10.1016/j.brainres.2020.146645

37. Heo JY, Nam M-H, Yoon HH, Kim J, Hwang YJ, Won W, et al. Aberrant Tonic Inhibition of Dopaminergic Neuronal Activity Causes Motor Symptoms in Animal Models of Parkinson's Disease. Current Biology. 2020; 30(2): 276-91. e9 https://doi.org/10.1016/j.cub.2019.11.079.

38. Blanchard JW, Bula M, Davila-Velderrain J, Akay LA, Zhu L, Frank A, et al. Reconstruction of the human blood-brain barrier in vitro reveals a pathogenic mechanism of APOE4 in pericytes. Nature Medicine. 2020: 1-12 https://doi.org/10.1038/s41591-020-0886-4.
39. Mozafari N, Ashrafi H, Azadi A. Targeted drug delivery systems to control neuroinflammation in central nervous system disorders. Journal of Drug Delivery Science and Technology. 2021: 102802 https://doi.org/10.1016/j.jddst.2021.102802.

40. Wang X, Chi J, Huang D, Ding L, Zhao X, Jiang $L$, et al. $\alpha$-synuclein promotes progression of Parkinson's disease by upregulating autophagy signaling pathway to activate the NLRP3 inflammasome. Experimental and Therapeutic Medicine. 2020; 19(2): 931-8 https://doi.org/10.3892/etm.2019.8297.

41. Wijeyekoon RS, Moore SF, Farrell K, Breen DP, Barker RA, Williams-Gray $\mathrm{CH}$. Cerebrospinal Fluid Cytokines and Neurodegeneration-Associated Proteins in Parkinson's Disease. Movement Disorders. 2020 https://doi.org/10.1002/mds.28015.

42. Tang P, Chong L, Li X, Liu Y, Liu P, Hou C, et al. Correlation between serum RANTES levels and the severity of Parkinson's disease. Oxidative Medicine and Cellular Longevity. 2014; 2014 https://doi.org/10.1155/2014/208408.

43. Hu Y, Zhang K, Zhang T, Wang J, Chen F, Qin W, et al. Exercise Reverses Dysregulation of T-Cell-Related Function in Blood Leukocytes of Patients With Parkinson's Disease. Frontiers in neurology. 2020; 10: 1389 https://doi.org/10.3389/fneur.2019.01389.

44. Mori MA, Delattre AM, Carabelli B, Pudell C, Bortolanza M, Staziaki PV, et al. Neuroprotective effect of omega-3 polyunsaturated fatty acids in the 6-OHDA model of Parkinson's disease is mediated by a reduction of inducible nitric oxide synthase. Nutritional neuroscience. 2018; 21(5): 341-51 https://doi.org/10.1080/1028415X.2017.12909 
28.

45. Belloli S, Morari M, Murtaj V, Valtorta S, Moresco R, Gilardi M. Translation Imaging in Parkinson's Disease: Focus on Neuroinflammation. 2020 https://doi.org/10.3389/fnagi.2020.00152.

46. Zhang L, Huang X, Guo T, Wang H, Fan H, Fang L. Study of Cinobufagin as a Promising Anticancer Agent in Uveal Melanoma Through Intrinsic Apoptosis Pathway. Frontiers in oncology. 2020; 10: 325 https://doi.org/10.3389/fonc.2020.00325.

47. Tompkins MM, Basgall EJ, Zamrini E, Hill WD. Apoptotic-like changes in Lewy-bodyassociated disorders and normal aging in substantia nigral neurons. The American journal of pathology. 1997; 150(1): 119.

48. Hartmann A, Hunot S, Michel PP, Muriel MP, Vyas S, Faucheux BA, et al. Caspase-3: a vulnerability factor and final effector in apoptotic death of dopaminergic neurons in Parkinson's disease. Proceedings of the National Academy of Sciences. 2000; 97(6): 2875-80

https://doi.org/10.1073/pnas.040556597.

49. Mogi M, Togari A, Kondo T, Mizuno Y, Komuro O, Kuno S, et al. Caspase activities and tumor necrosis factor receptor R1 (p55) level are elevated in the substantia nigra from parkinsonian brain. Journal of neural transmission. 2000; 107(3): 335-41 https://doi.org/10.1007/s007020050028.

50. Silva J, Alves C, Freitas R, Martins A, Pinteus S, Ribeiro $\mathrm{J}$,et al. Antioxidant and Neuroprotective Potential of the Brown Seaweed Bifurcaria bifurcate in an in vitro Parkinson's Disease Model. Marine drugs. 2019; $\quad 17(2)$ : $\quad 85$ https://doi.org/10.3390/md17020085.

51. Motawi TK, Sadik NAH, Hamed MA, Ali
SA, Khalil WKB, Ahmed YR. Potential therapeutic effects of antagonizing adenosine $\mathrm{A} 2 \mathrm{~A}$ receptor, curcumin, and niacin in rotenone-induced Parkinson's disease mice model. Molecular and Cellular Biochemistry. 2020; 465(1): 89-102 https://doi.org/10.1007/s11010-019-03670-0 https://doi.org/10.1007/s11010-019-03670-0.

52. Fiskum G, Starkov A, Polster BM, Chinopoulos C. Mitochondrial mechanisms of neural cell death and neuroprotective interventions in Parkinson's disease. ANNALS-NEW YORK ACADEMY OF SCIENCES. 2003; 991: 111-9 https//:doi.org/10.1111/j.17496632.2003.tb07469.x.

53. Sabnis RW. Novel N-Heteroaryl Quinazolin2-amine Derivatives as LRRK2 Inhibitors for Treating Parkinson's Disease. ACS Publications; 2021.

54. Zheng L, Bernard-Marissal N, Mullan N, D'Amico D, Auwerx J, Moore DJ, et al. Parkin functionally interacts with PGC- $1 \alpha$ to preserve mitochondria and protect dopaminergic neurons. Human molecular $\begin{array}{lll}\text { genetics. } & 2017 ; \quad 26(3): \quad 582-98\end{array}$ https://doi.org/10.1093/hmg/ddw418.

55. Meng Y, Qiao H, Ding J, He Y, Fan H, Li C, et al. Effect of Parkin on methamphetamine-induced $\quad \alpha$-synuclein degradation dysfunction in vitro and in vivo. Brain and Behavior. 2020; 10(4): e01574 https://doi.org/10.1002/brb3.1574.

56. Lee J-W, Cannon JR. LRRK2 mutations and neurotoxicant susceptibility. Experimental Biology and Medicine. 2015;240(6):752-9 https://doi.org/10.1177/1535370215579162.

57. Sanders LH, Laganière J, Cooper O, Mak SK, $\mathrm{Vu}$ BJ, Huang YA, et al. LRRK2 mutations cause mitochondrial DNA damage in iPSC- 
derived neural cells from Parkinson's disease patients: reversal by gene correction. Neurobiology of disease. 2014; 62: 381-6 https://doi.org/10.1016/j.nbd.2013.10.013.

58. Iaccarino C, Crosio C, Vitale C, Sanna G, Carrì MT, Barone P. Apoptotic mechanisms in mutant LRRK2-mediated cell death. Human molecular genetics. 2007; 16(11): 1319-26 https://doi.org/10.1093/hmg/ddm080.

59. Perier C, Bender A, García-Arumí E, Melia MJ, Bové J, Laub C, et al. Accumulation of mitochondrial DNA deletions within dopaminergic neurons triggers neuroprotective mechanisms. Brain. 2013; 136(8): 2369-78 https://doi.org/10.1093/brain/awt196.

60. Reeve AK, Krishnan KJ, Elson JL, Morris CM, Bender A, Lightowlers RN, et al. Nature of mitochondrial DNA deletions in substantia nigra neurons. The American Journal of Human Genetics. 2(1)82;008 35-228: https://doi.org/10.1016/j.ajhg.2007.09.018.

61. Liu C-Y, Lee C-F, Hong C-H, Wei Y-H. Mitochondrial DNA mutation and depletion increase the susceptibility of human cells to apoptosis. Mitochondrial Pathogenesis: Springer; 2004. p. 133-45 https://doi.org/10.1007/978-3-662-410882_14.

62. Dölle C, Flønes I, Nido GS, Miletic H, Osuagwu N, Kristoffersen S, et al. Defective mitochondrial DNA homeostasis in the substantia nigra in Parkinson disease. Nature communications. 2016;7(1):1-11 https//:doi.org/10.1038/ncomms13548.

63. Zeng X-S, Geng W-S, Jia J-J. Neurotoxininduced animal models of Parkinson's disease: pathogenic mechanism and assessment. ASN Neuro. $\quad 2018 ; \quad 10$ : 1759091418777438 https://doi.org/10.1177/1759091418777438.
64. Gonçalves DF, Courtesy AA, Hartmann DD, da Rosa PC, Oliveira DM, Soares FA, et al. 6Hydroxydopamine induces different mitochondrial bioenergetics response in brain regions of rat. Neurotoxicology. 2019; 70: 111 https://doi.org/10.1016/j.neuro.2018.10.005.

65. Kheradmand A ,Nayebi AM, Jorjani M, Haddadi R. Effect of WR-1065 on 6hydroxydopamine-induced catalepsy and IL-6 level in rats. Iranian journal of basic medical sciences. 2016; 19(5): 490.

66. Lai C-L, Lu C-C, Lin H-C, Sung Y-F, Wu Y$\mathrm{P}$, Hong $\mathrm{J}-\mathrm{S}$, et al. Valproate is protective against 6-OHDA-induced dopaminergic neurodegeneration in rodent midbrain: $A$ potential role of BDNF up-regulation. Journal of the Formosan Medical Association. 2019; 118(1): 420-8 https://doi.org/10.1016/j.jfma.2018.06.017.

67. Prajapati SK, Garabadu D, Krishnamurthy S. Coenzyme Q10 prevents mitochondrial dysfunction and facilitates pharmacological activity of atorvastatin in 6-OHDA induced dopaminergic toxicity in rats. Neurotoxicity research. 2017; 31(4): 478-92 https://doi.org/10.1007/s12640-016-9693.6-

68. Laloux C, Perrault M, Lecointe C, Devos D, Bordet R. Differential susceptibility to the PPAR- $\gamma$ agonist pioglitazone in 1-methyl-4phenyl-1, 2, 3, 6-tetrahydropyridine and 6hydroxydopamine rodent models of Parkinson's disease. Pharmacological Research. 2012; 65(5): 514-22 https://doi.org/10.1016/j.phrs.2012.02.008.

69. Hwang O. Role of oxidative stress in Parkinson's disease. Experimental neurobiology. 2013;22(1):11-7 https://doi.org/10.5607/en.2013.22.1.11.

70. Lee KJ, Shim I, Sung JH, Hong JT, sup Kim I, Cho CB. Striatal glutamate and GABA after 
high-frequency subthalamic stimulation in the parkinsonian rat. Journal of Korean Neurosurgical Society. 2017; 60(2): 138 https://doi.org/10.3340/jkns.2016.0202.020.

71. Larramendy C, Taravini IR, Saborido MD, Ferrario JE, Murer MG, Gershanik OS. Cabergoline and pramipexole fail to modify already established dyskinesias in an animal model of parkinsonism. Behavioral brain research. 2008; 194(1): 44-51 https://doi.org/10.1016/j.bbr.2008.06.021.

72. Kuruvilla KP, Nandhu M, Paul J, Paulose C. Oxidative stress-mediated neuronal damage in the corpus striatum of 6-hydroxydopamine lesioned Parkinson's rats: Neuroprotection by serotonin, GABA and bone marrow cells supplementation. Journal of the Neurological Sciences. $\quad 20 \quad 7-31:(2-1) 331 ; 13$ https://doi.org/10.1016/j.jns.2013.04.020.

73. Zhengli C, Shi R, Cheng A, Zeng W, Liu W, $\mathrm{Xia} \mathrm{Y}$, et al. Clioquinol improves motor and non-motor deficits in MPTP-induced monkey model of Parkinson's disease through AKT/mTOR pathway. Aging. 2 33-9515:(10)12;020 https://doi.org/10.18632/aging.103225.

74. Langston JW, Forno LS, Rebert CS, Irwin I. Selective nigral toxicity after systemic administration of 1-methyl-4-phenyl-1, 2, 5, 6-tetrahydropyran (MPTP) in the squirrel monkey. Brain research 4-390:(2)292;1984 . https://doi.org/10.1016/0006-8993(84)907777.

75. Langston JW. The MPTP story. Journal of Parkinson's disease. 2017;7(s1):S11-S9 https://doi.org/10.3233/JPD-179006.

76. Zhang J-J, Shi X-R, Lv W-W, Zhou X-L, Sun Y-D, Li B-Y, et al. Neuroprotective Effects of Lindleyin on Hydrogen Peroxide-Induced Cell Injury and MPTP-Induced Parkinson's
Disease in C57BL/6 Mice. Evidence-Based Complementary and Alternative Medicine. 2020;2020 https://doi.org/10.1155/2020/2938432.

77. Rahimi Jaberi K, Safari M, Semnani V, Sameni HR, Zarbakhsh S, Ghahari L. Caffeic Acid Phenethyl Ester with Mesenchymal Stem Cells Improve Behavioral and Histopathological Changes in the Rat Model of Parkinson'Disease. Basic and Clinical Neuroscience. 2020:0https://doi.org/10.32598/bcn.2021.1398.1.

78. Fornai F, Lenzi P, Ferrucci M, Lazzeri G, Di Poggio AB, Natale $G$, et al. Occurrence of neuronal inclusions combined with increased nigral expression of $\alpha$-synuclein within dopaminergic neurons following treatment with amphetamine derivatives in mice. Brain research bulletin. 2005; 65(5): 405-13 https://doi.org/10.1016/j.brainresbull.2005.02. 022.

79. Shimoji M, Zhang L, Mandir AS, Dawson VL, Dawson TM. Absence of inclusion body formation in the MPTP mouse model of Parkinson's disease. Molecular brain research. 2005; 134(1): 103-8 https://doi.org/10.1016/j.molbrainres.2005.01. 012.

80. Vivacqua G, Biagioni F, Busceti CL, Ferrucci M, Madonna M, Ryskalin L, et al. Motor Neurons Pathology After Chronic Exposure to MPTP in Mice. Neurotoxicity Research. 2020; 37(2):

298-313 https://doi.org/10.1007/s12640-019-00121-y.

81. Hou L, Huang R, Sun F, Zhang L, Wang Q. $\mathrm{NADPH}$ oxidase regulates paraquat and maneb-induced dopaminergic neurodegeneration through ferroptosis. Toxicology. 2019; 417: 64-73 https://doi.org/10.1016/j.tox.2019.02.011. 
82. Joseph TP, Jagadeesan N, Sai LY, Lin SL, Sahu S, Schachner M. Adhesion Molecule L1 Agonist Mimetics Protect Against the Pesticide Paraquat-Induced Locomotor Deficits and Biochemical Alterations in Zebrafish. Frontiers in Neuroscience. 2020 https://doi.org/10.3389/fnins.2020.00458.

83. Takizawa M, Komori K, Tampo Y, Yonaha M. Paraquat-induced oxidative stress and dysfunction of cellular redox systems including antioxidative defense enzymes glutathione peroxidase and thioredoxin reductase. Toxicology in Vitro. 2007; 21(3): 355-63

https://doi.org/10.1016/j.tiv.2006.09.003.

84. Chen Y-B, Wang Y-Q, Wu J-R, Cui Y-L. A novel idea for establishing Parkinson's disease mouse model by intranasal administration of paraquat .Neurological Research. 2021; 43(4): 267-77

https://doi.org/10.1080/01616412.2020.18475 42.

85. Chia SJ, Tan E-K, Chao Y-X. Historical perspective: Models of Parkinson's disease. International journal of molecular sciences. 2020; 21(7): 2464 https://doi.org/10/3390.ijms21072464.

86. Johnson ME, Bobrovskaya L. An update on the rotenone models of Parkinson's disease: their ability to reproduce the features of clinical disease and model gene-environment interactions. Neurotoxicology. 2015; 46: 10116

https://doi.org/10.1016/j.neuro.2014.12.002.

87. Han Y, Wang T, Li C, Wang Z, Zhao Y, He $\mathrm{J}$, et al. Ginsenoside $\mathrm{Rg} 3$ exerts a neuroprotective effect in rotenone-induced Parkinson's disease mice via its anti-oxidative properties. European Journal of Pharmacology. 2021:1744 13 https://doi.org/10.1016/j.ejphar.2021.174413.
88. Worth AJ, Basu SS, Snyder NW, Mesaros C, Blair IA. Inhibition of neuronal cell mitochondrial complex I with rotenone increases lipid $\beta$-oxidation, supporting acetylcoenzyme A levels. Journal of Biological Chemistry. 2014; 289(39): 26895-903 https://doi.org/10.1074/jbc.M114.591354.

89. Betarbet R, Sherer TB, MacKenzie G, Garcia-Osuna M, Panov AV, Greenamyre JT. Chronic systemic pesticide exposure reproduces features of Parkinson's disease. Nature neuroscience. 2000; 3(12): 1301-6 https://doi.org/10.1038/81834.

90. Greenamyre JT, Cannon JR, Drolet R, Mastroberardino P-G. Lessons from the rotenone model of Parkinson's disease. Trends in pharmacological sciences. 2010; 31(4): 141 https://doi.org/10.1016/j.tips.20.09.12.006 